\title{
Silicon Microspheres for Integrated Photonics
}

\author{
Ulas Kemal Ayaz, ${ }^{\dagger}$ Adnan Kurt, and Ali Serpengüzel* \\ Koç University, Microphotonics Research Laboratory, Physics Department, \\ Rumeli Feneri Yolu, Sariyer, Istanbul 34450 Turkey
}

\begin{abstract}
We have experimentally studied the electrical and optical properties of a silicon microsphere with a radius of 500 microns and a refractive index of 3.48 in the near-infrared. The silicon microsphere shows an MSM Schottky diode behavior. The morphology dependent resonances are spaced by $0.2 \mathrm{~nm}$, which correlates well with the estimated mode spacing. The silicon microsphere heralds the way for a novel family of active microphotonic devices.
\end{abstract}

Keywords: Channel dropping filter, dielectric microsphere, evanescent coupling, integrated optoelectronics, microsphere resonator, morphology dependent resonances, optical resonance, optical coupler, optical fiber, whispering gallery modes.

\section{INTRODUCTION}

With the development of optoelectronics, metal interconnections are no longer limiting factor for the performance of electronic systems. Replacing the metal interconnections by optical interconnections could provide low power dissipation, low latencies, and high-bandwidths. Such optical interconnections rely on the integration of microphotonics with microelectronics. Microsphere resonators are ideal microphotonic building blocks due to their small volumes and high quality factors. There have been various photonic applications of microspheres in the ultraviolet (UV), visible and near-infrared (IR) communication bands $\left[^{1}\right]$. Morphology dependent resonances (MDR's), i.e., whispering gallery modes (WGM's), or simply optical resonances of dielectric microspheres provide the necessary optical feedback for applications in spectroscopy, laser science, and optical communications. Microlasers, optical couplers, and optical filters are some of the applications of microspheres. Low threshold lasing from rare earth doped silica microspheres $\left[{ }^{2}\right]$, polymer microsphere lasers $\left[{ }^{3}\right]$ and Raman lasers have been demonstrated. Strain tunable microsphere oscillators $\left[{ }^{4}\right]$, add-drop filters $\left[^{5}\right]$, and thermooptical switching $\left[\left[^{6}\right]\right.$ have been realized for the frequency control in optical communications.

Microsphere resonators are uniquely applicable in compact optoelectronic devices in wavelength division multiplexing (WDM) applications $\left[^{7}\right]$. WDM provides the advantage of increasing the bandwidth of communication network. In these lightwave circuits, microspheres can be used as compact channel dropping filters. Recently, we have observed elastic light scattering in silicon microspheres in the near-infrared $\left[{ }^{8}\right]$. In this paper, we are investigating the electrooptical response of these silicon microspheres.

\section{MORPHOLOGY DEPENDENT RESONANCES}

A physical interpretation of MDR's is based on the propagation of rays around the inside surface of the microsphere,

* Correspondence should be addressed to Ali Serpengüzel, aserpenguzel@ku.edu.tr; phone: + 90 (212) 338-1312; fax: + 90 (212) 3381547, http://home.ku.edu.tr/ aserpenguzel, http://microphotonics.ku.edu.tr, Koç University, Microphotonics Research Laboratory, Physics Department, Rumeli Feneri Yolu, Sariyer, Istanbul 34450 Turkey. ${ }^{\dagger}$ Ulas Kemal Ayaz is currently with Polytechnic University, Department of Mechanical, Aerospace, and Manufacturing Engineering, Six MetroTech Center, Brooklyn NY 11201, USA.

Optoelectronic Integrated Circuits IX, edited by Louay A. Eldada, El-Hang Lee Proc. of SPIE Vol. 6476, 647606, (2007) · 0277-786X/07/\$18 - doi: 10.1117/12.706279 
confined by an almost total internal reflection (TIR) $\left[{ }^{9}\right]$. The rays approach the interior surface of the microsphere at an angle beyond the critical angle and thus experience an almost total internal reflection (TIR). The light rays follow the interior surface of the microsphere, and return to their respective entrance points at the same phase and start to propagate in the interior surface of the microsphere all over again. This basically is the physical interpretation of the MDR's for a microsphere. The light, after circumnavigating the interior surface of the microsphere, returns to its entrance point and interferes with itself. At resonant wavelengths, the interference is constructive and the elastically scattered light from the microsphere reaches to peak intensities. Respective to the peaks in elastically scattered light, the intensity of transmitted light through fiber experiences associated dips in intensity $\left[{ }^{10}\right]$. MDR's satisfy resonance conditions for specific values of the size parameter $x=2 \pi a / \lambda$, where $a$ is radius of the sphere and $\lambda$ is the vacuum wavelength of light $\left[{ }^{11}\right]$.

The MDR's of a microsphere are analyzed by the localization principle $\left[{ }^{12}\right]$ and the generalized Lorenz-Mie theory (GLMT) $\left[{ }^{13}\right]$. The angular mode number (n) gives the number of maxima between $0 \mathrm{o}$ and $180 \mathrm{o}$ degrees in the polar angular distribution of the energy of the MDR. The radial mode order (l) indicates the number of maxima in the internal electric field distribution in the radial direction. Each MDR of the microsphere also has an azimuthal angular dependence from 0 to 360 degrees, which is labeled with an azimuthal mode number. However, for spheres, MDR's differing only in azimuthal mode number have identical resonance frequencies.

The greatest impediment to the use of microsphere resonators has been the difficulty of coupling the light into and out of the microspheres efficiently. Light can be coupled into the microsphere by various types of coupling devices: side-polished optical fibers $\left[{ }^{14}\right]$, prisms $\left[{ }^{15}\right]$ and tapered optical fibers $\left[{ }^{16}\right]$. The principle of these devices is based on providing efficient energy transfer to the resonant circular TIR guided wave in the microsphere resonator through the evanescent field. There are two main conditions to be satisfied to achieve efficient coupling: phase synchronism and significant overlap of the two waves modeling the MDR mode and the coupler mode. The coupling is based on the fact that the modal field of the guided mode extends beyond the core-cladding interface.

\section{ELECRO-OPTICAL EFFECT IN SILICON}

In the presence of free carriers in silicon, both the refractive index and the absorption of silicon can be changed using carrier injection or depletion. The refractive index changes due to free carrier accumulation is given by the Kramers-Kronig analysis $\left[{ }^{17}\right]$ :

$$
\begin{aligned}
& \Delta \mathrm{n}_{\mathrm{e}}=-8.8 \times 10^{-22} \Delta \mathrm{N}_{\mathrm{e}} \\
& \Delta \mathrm{n}_{\mathrm{h}}=-8.5 \times 10^{-18}\left(\Delta \mathrm{N}_{\mathrm{h}}\right)^{0.8}
\end{aligned}
$$

where $\Delta \mathrm{n}_{\mathrm{e}}$ and $\Delta \mathrm{n}_{\mathrm{h}}$ are the changes in the refractive index resulting from change in the free electron carrier concentrations and change in the free hole concentrations. $\Delta \mathrm{N}_{\mathrm{e}}$ and $\Delta \mathrm{N}_{\mathrm{h}}$ are the free electron and free hole concentrations, respectively.

\section{ELECTRICAL MEASUREMENTS}

By placing two probes to the poles of the sphere, we made the metal-silicon-metal (MSM) contact. Figure 1 shows the schematic diagram of the MSM contact that was used in our experiments. The I-V characteristics illustrated in figure 2 support that the diode is two Schottky diodes connected back to back. The graph is not fully symmetric, due to the non-symmetric contact. The contact resistance differences are not significant. 


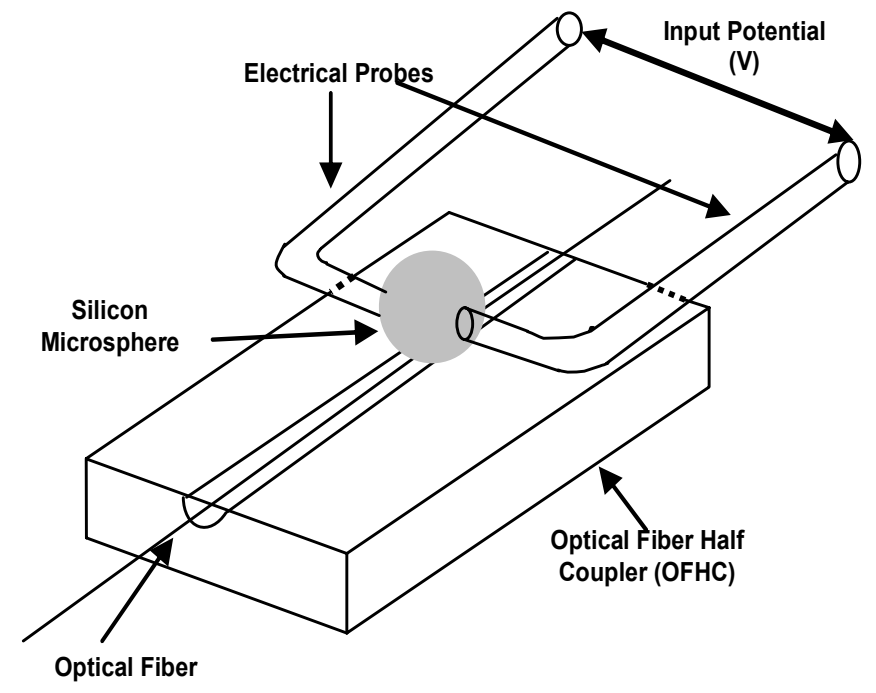

Fig.1. Illustration of electrical input to the microsphere over the OFHC.

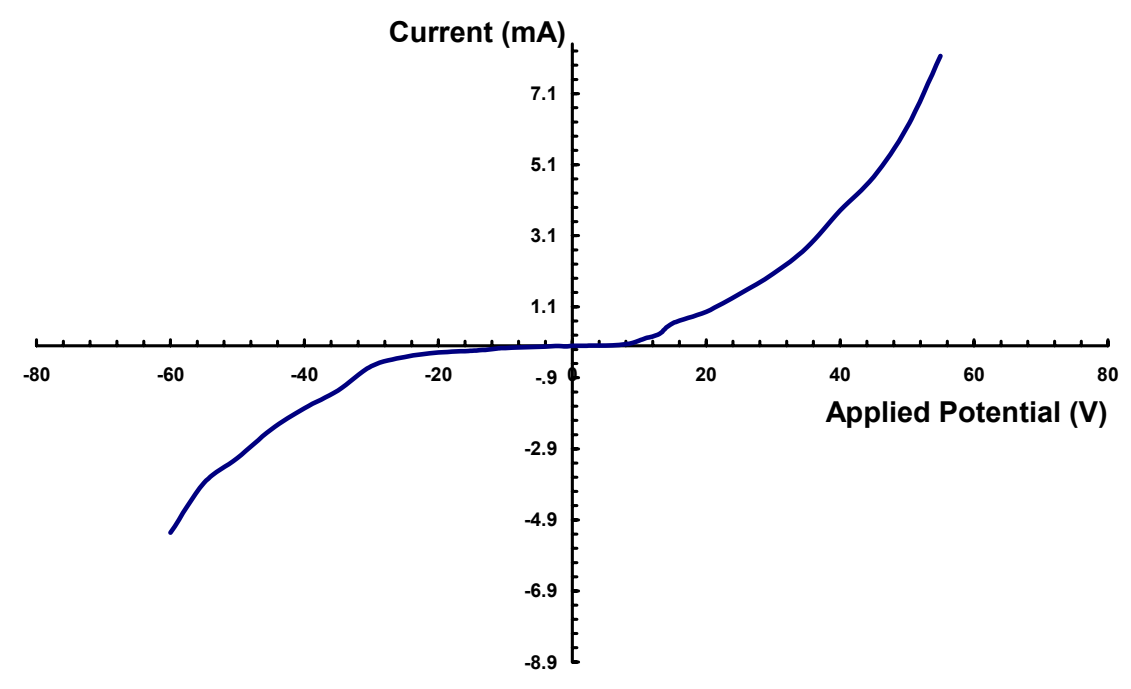

Fig.2. The I-V characteristics of the experimental metal-silicon-metal (MSM) contact.

\section{OPTICAL MEASUREMENTS}

Light is coupled to the silicon microsphere by using an optical fiber half coupler (OFHC). Figure 3 shows the optical geometry of the microsphere and the OFHC. The OFHC is manufactured by polishing the optical fiber that is glued in a silica substrate. OFHC is fabricated from $1500 \mathrm{~nm}$ standard single mode fiber (SMF). The cladding is polished down to approach the evanescent field of the optical fiber. The silicon microsphere used in the experiment has a radius $\mathrm{a}=500 \mu \mathrm{m}$ and refractive index $\mathrm{n}=3.48$. The silicon microsphere is placed on the interaction region of the OFHC. The output of a distributed feedback (DFB) laser is coupled to the optical fiber. Temperature tuning of the DFB laser is achieved by using a laser diode controller (LDC). The transmitted light is observed using an InGaAs optical power/wave head connected to an optical multimeter (OMM). The elastically scattered light from the 
microsphere is collected through a microscope objective and detected by the InGaAs photodiode. The microsphere was held in position by electrical probes from the poles, which were also used for electrical input. A typical spectrum is shown in Fig. 5.

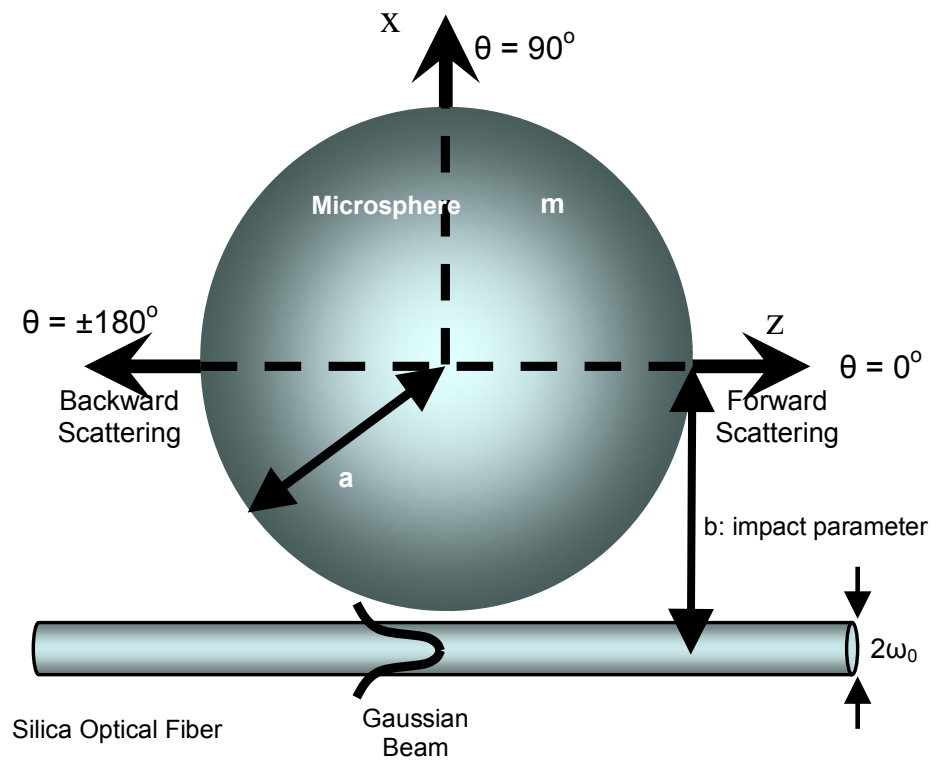

Fig.3. Schematic illustration of the optical fiber excitation of the silicon microsphere.

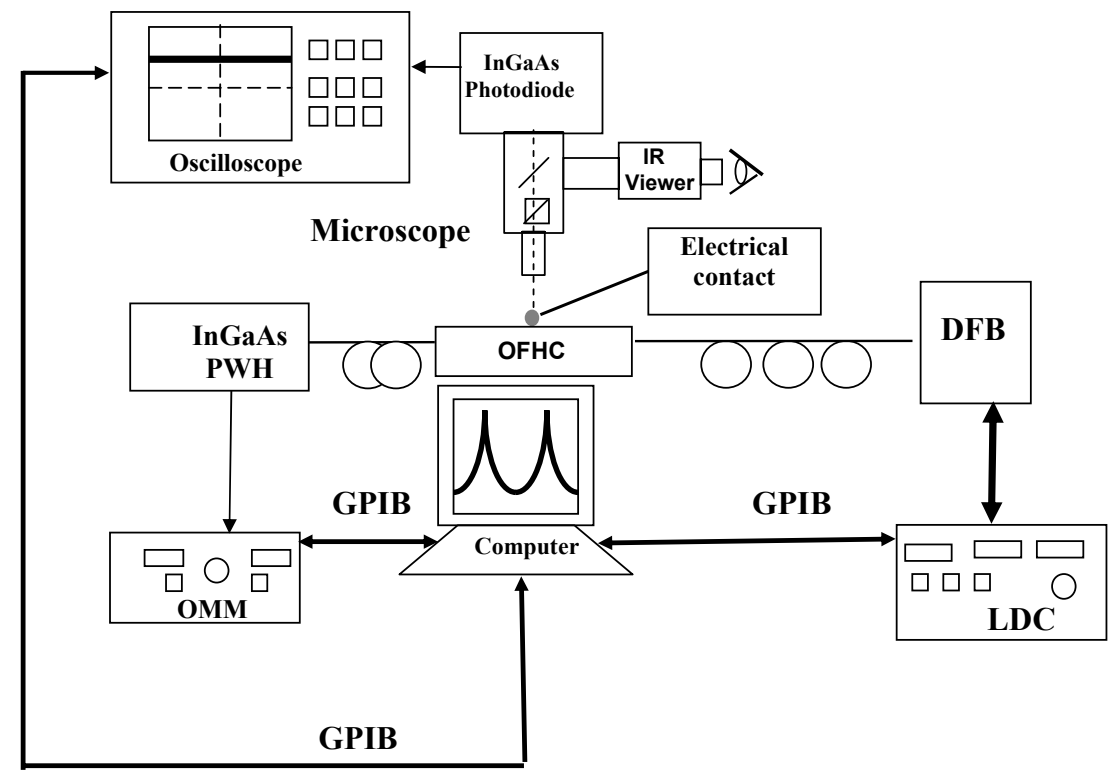

Fig.4. The schematic of the experimental setup. 


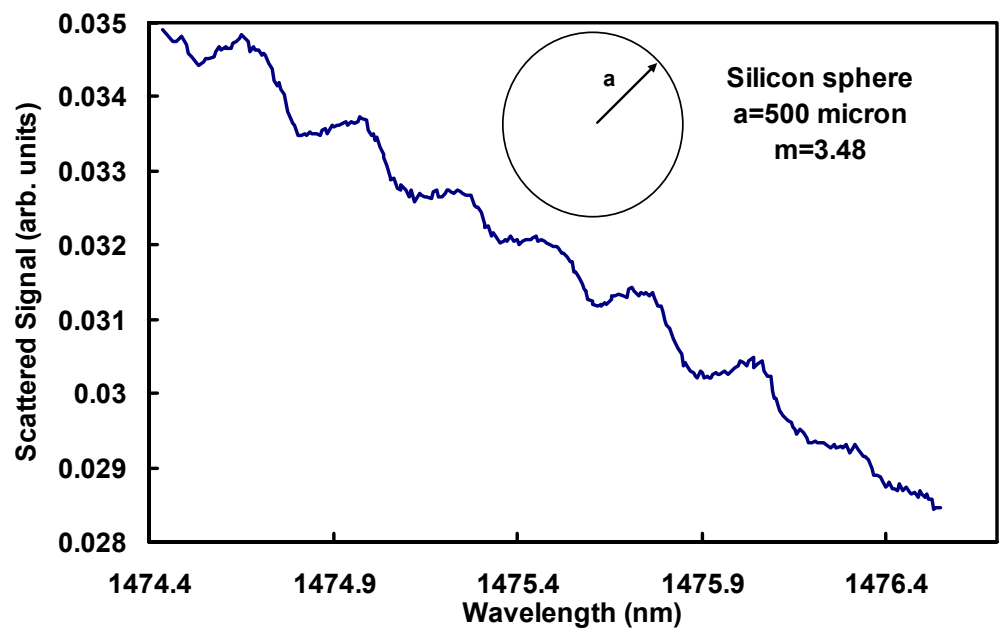

Fig.5. A typical spectrum from the silicon microsphere.

\section{CONCLUSIONS}

We have experimentally observed the elastic scattered signal from a silicon microsphere in the near-IR at a wavelength of $1475 \mathrm{~nm}$. The mode spacing $(\Delta \lambda)$, i.e. wavelength difference between consecutive mode numbers $(\mathrm{n})$ with the same mode order (1), is measured to be $0.27 \mathrm{~nm}$. Moreover, we have measured the IV characteristics of the silicon microsphere sandwiched between metal probes. The MSM structure exhibits two back to back Schottky diode response. These observations herald novel active silicon microsphere based optoelectronic device applications. Possible wavelength division multiplexing (WDM) applications include optoelectronic devices for filtering, detection, modulation, and switching.

\section{ACKNOWLEDGMENTS}

We would like to acknowledge the partial support of this research by the European Commission Grant No: FP6-IST511616: PHOREMOST and FP6-IST-003887 NEMO.

\section{REFERENCES}

1. S. M. Spillane, T. J. Kippenberg, and K. J. Vahala, "Ultralow-threshold Raman laser using a spherical dielectric microcavity", Nature, vol. 415, pp. 621-623, Feb. 2002.

2. V. Lefèvre-Seguin and S. Haroche, "Towards cavity-QED experiments with silica microspheres”, Mat. Sci. Eng., vol. B48 pp. 53-58, 1997.

3. M. Kuwata-Gonokami and K. Takeda, "Polymer whispering gallery mode lasers", Opt. Mat., vol. 9, pp. 12-17, Jan. 1998.

4. V. S. Ilchenko, P. S. Volikov, V. L. Velichansky, F. Treussart, V. Lefèvre-Seguin, J.-M. Raimond, and S. Haroche, "Strain-tunable high-Q optical microsphere resonator", Optics Comm., vol.145, pp. 86-90, Jan. 1998.

5. M. Cai, G. Hunziker, and K. J. Vahala, "Fiber-optic add-drop device based on a silica microsphere- whispering gallery mode system," IEEE Photon. Technol. Lett., vol. 6, no. 6, pp. 686-687, June 1999.

6. H. C. Tapalian, J.-P. Laine, and P. A. Lane, "Thermooptical switches using coated microsphere resonators", IEEE Photon. Technol. Lett., vol. 14, no. 8, pp. 1118-1120, Aug. 2002.

7. A. Serpengüzel, S. Arnold, G. Griffel, and J. A. Lock, "Enhanced coupling to microsphere resonances with optical fibers," J. Opt. Soc. Am. B., vol. 14, no. 4, pp. 790-795, Apr. 1997.

8. Y.O. Yilmaz, A. Demir, A. Kurt, and A. Serpengüzel, "Optical Channel Dropping with a Silicon Microsphere," IEEE Photon. Technol. Lett., 17, 1662-1664, 2005.

9. P. Barber, and R. K. Chang, Optical Effects Associated with Small Particles, Singapore: World Scientific, 1988.

10. P. Barber, and S. C. Hill, Light Scattering by Particles: Computational Methods. Singapore: World Scientific, 1990.

11. M. Pelton, and Y. Yamamato, "Ultralow threshold laser using a single quantum dot and a microsphere cavity," Phys. Rev. A, vol. 59, pp. 2418-2421, Mar. 1999.

12. H.M. Nussenzveig, "Diffraction Effects in Semiclassical Scattering," Cambridge University Press, 1992 
13. G. Griffel, S. Arnold, D. Taskent, A. Serpengüzel, J. Connolly, and N. Morris, "Morphology Dependent Resonances of a Microsphere, Optical Fiber System," Opt. Lett., vol. 21, no. 10, pp. 695-697, 1996.

14. A. Serpengüzel, S. Arnold, and G. Griffel, "Excitation of Resonances of Microspheres on an Optical Fiber", Optics Letters, Vol. 20, No. 7 , pp. 654-656, April 1995

15. M. L. Gorodetsky and V. S. Ilchenko, "High- $Q$ optical whispering gallery microresonators: precession approach for spherical mode analysis and emission patterns," Opt. Commun. 113, 133-143 (1994).

16. J. C. Knight, G. Cheung, F. Jacques, and T. A. Birks, "Phase matched excitation of whispering gallery modes by a fiber taper," Opt. Lett., Vol. 22, pp. 1129-1131, Aug. 1997.

17. G. T. Reed and A. P. Knights: "Silicon Photonics; An Introduction" John Wiley and Sons Ltd. West Sussex, (2004). 